\title{
Site-specific somatic mitochondrial DNA point mutations in patients with thymidine phosphorylase deficiency
}

\author{
Yutaka Nishigaki, ${ }^{1}$ Ramon Martí, ${ }^{1}$ William C. Copeland, ${ }^{2}$ and Michio Hirano ${ }^{1}$ \\ ${ }^{1}$ Department of Neurology, Columbia University College of Physicians and Surgeons, New York, New York, USA \\ ${ }^{2}$ Laboratory of Molecular Genetics, National Institute of Environmental Health Sciences, National Institute of Health, \\ Research Triangle Park, North Carolina, USA
}

\begin{abstract}
Mitochondrial neurogastrointestinal encephalomyopathy (MNGIE) is an autosomal recessive disorder caused by loss-of-function mutations in the gene encoding thymidine phosphorylase (TP). This deficiency of TP leads to increased circulating levels of thymidine (deoxythymidine, dThd) and deoxyuridine (dUrd) and has been associated with multiple deletions and depletion of mitochondrial DNA (mtDNA). Here we describe 36 point mutations in mtDNA of tissues and cultured cells from MNGIE patients. Thirty-one mtDNA point mutations $(86 \%)$ were T-to-C transitions, and of these, 25 were preceded by 5 -AA sequences. In addition, we identified a single base-pair mtDNA deletion and a TT-to-AA mutation. Next-nucleotide effects and dislocation mutagenesis may contribute to the formation of these mutations. These results provide the first demonstration that alterations of nucleoside metabolism can induce multiple sequence-specific point mutations in humans. We hypothesize that, in patients with TP deficiency, increased levels of dThd and dUrd cause mitochondrial nucleotide pool imbalances, which, in turn, lead to mtDNA abnormalities including site-specific point mutations.
\end{abstract}

J. Clin. Invest. 111:1913-1921 (2003). doi:10.1172/JCI200317828.

\section{Introduction}

Mitochondrial neurogastrointestinal encephalomyopathy (MNGIE) is characterized clinically by onset in the second to fifth decade of life, ptosis, progressive external ophthalmoparesis, gastrointestinal dysmotility, cachexia, peripheral neuropathy, myopathy, and leukoencephalopathy $(1,2)$. The disease is associated with multiple deletions and depletion of mitochondrial DNA (mtDNA), which have been ascribed to a defect in intergenomic communication between the nuclei and mitochondria $(1,3,4)$. We mapped the disease locus to chromosome 22q13.32-qter (5), and identified loss-of-function mutations in the encoding thymidine phosphorylase (TP; E.C.2.4.2.4) gene as the cause of MNGIE (6). TP activity in buffy coat samples

Received for publication March 12, 2002, and accepted in revised form May 25, 2002.

Address correspondence to: Michio Hirano, Columbia University College of Physicians and Surgeons, 630 West 168th Street, Physicians and Surgeons 4-443, New York, New York 10032, USA. Phone: (212) 305-1048; Fax: (212) 305-3986; E-mail:mh29@columbia.edu.

Conflict of interest: The authors have declared that no conflict of interest exists.

Nonstandard abbreviations used: mitochondrial neurogastrointestinal encephalomyopathy (MNGIE); mitochondrial DNA (mtDNA); thymidine phosphorylase (TP); thymidine (dThd); deoxyuridine (dUrd); cytochrome $c$ oxidase (COX); restriction fragment length polymorphism (RFLP); hypervariable segment (HVS); displacement loop (D-loop); deoxynucleoside 5'-triphosphate (dNTP); deoxyguanosine monophosphate (dGMP); deoxythymydine triphosphate (dTTP); polymerase $\gamma(\operatorname{pol} \gamma)$; deoxythymidine monophosphate (dTMP); deoxyuridine triphosphate (dUTP); deoxyadenosine monophosphate (dAMP). from patients is less than $10 \%$ of the control mean, indicating that loss-of-function mutations in TP cause the disease (6).

TP deficiency alters the metabolism of thymidine (deoxythymidine, dThd) (7) and deoxyuridine (dUrd); therefore, we have hypothesized that increased levels of dThd and dUrd cause imbalances of mitochondrial nucleotide pools that in turn lead to mtDNA abnormalities. In support of our hypothesis is the observation that mitochondrial alterations are present in skeletal muscle; however, this tissue does not express TP (1). This "muscle paradox" indicates that TP deficiency causes mtDNA alterations indirectly through abnormal nucleoside metabolism.

\section{Methods}

Patients. We previously identified homozygous or compound-heterozygous TP gene mutations in 13 MNGIE patients (six families) from diverse ethnic groups (6), including Ashkenazi Jewish (MNGIE family 1 [MN1]), German American (MNGIE family 2 [MN2]) (2), Puerto Rican (MNGIE family 3 [MN3]), African American (MNGIE family 4 [MN4]), Puerto Rican (MNGIE family 6 [MN6]), and mixed European (MNGIE family 7 [MN7]). The number following MN refers to a family; individuals were identified by a second number (e.g., MN1-1).

Cell culture. We cultured skin fibroblasts from six MNGIE patients and six age-matched controls. We plated $10^{5}$ fibroblasts from each patient or control in Petri dishes (10 cm in diameter) with $10 \mathrm{ml}$ MEM (Invitrogen Corp., Carlsbad, California, USA) plus 15\% FBS, $1 \mathrm{mM}$ pyruvate, $100 \mu \mathrm{M}$ nonessential amino 
acids, $2 \mathrm{mM}$ L-glutamine, and $1 \times$ MEM vitamin solution (Invitrogen Corp.). We grew the cells at $37^{\circ} \mathrm{C}$ in $5 \% \mathrm{CO}_{2}$. DNA was purified from passage three (MN4-2 and MN7-2), passage four (MN4-2, MN4-3, MN7-1, and MN7-2), or passage five (MN1-4 and MN3-1) cells. Determination of cytochrome $c$ oxidase and citrate synthase. Skin fibroblasts of six patients and three controls were cultured in the conditions described above. Confluent cells were trypsinized and washed with PBS, and the pellets were resuspended in reaction buffer (50 mM potassium phosphate, $\mathrm{pH}$ 7.0). Cells were lysed with one cycle of freezing and thawing. Cell lysates were treated with $1.3 \mathrm{mM}$ lauryl maltoside prior to cytochrome $c$ oxidase (COX) measurements and with $0.5 \%$ Triton X-100 prior to citrate synthase determinations. Established methods were used to measure activities of COX and citrate synthase (8). The protein content was determined using the bicinchoninic acid method (9).

Measurement of $d U r d$. Plasma dUrd was measured using a previously described HPLC method with modifications $(10,11)$. Plasma was treated with perchloric acid ( $0.5 \mathrm{M}$ final concentration) and centrifuged to remove the precipitated protein. Fifty microliters of the supernatant was injected into an Alliance HPLC apparatus from Waters Corp. (Milford, Massachusetts, USA) using an Alltima reverse-phase column (C18 NUC, $100 \AA, 5 \mu \mathrm{m}, 250 \times 4.6 \mathrm{~mm})$ preceded by an Alltima guard column $(\mathrm{C} 18,5 \mu \mathrm{m}, 7.5 \times 4.6 \mathrm{~mm})$ (Alltech Associates Inc., Deerfield, Illinois, USA). Injected samples were eluted over 110 minutes through a buffered methanol-gradient mobile phase. Eluent A was $20 \mathrm{mM}$ potassium phosphate, $\mathrm{pH} 5.6$, and eluent $\mathrm{B}$ was 20 $\mathrm{mM}$ potassium phosphate and $60 \%$ methanol, $\mathrm{pH}$ 5.6. The elution was performed with a constant flow rate of $1.5 \mathrm{ml} / \mathrm{min}$ as follows: time 0 to 5 minutes, $100 \%$ eluent A; 5 minutes to 92 minutes, $100 \%$ to $0 \%$ eluent A; 92 minutes to 93 minutes, 0 to $100 \%$ eluent A; 93 minutes to 110 minutes, constant $100 \%$ eluent $A$. The absorption of the eluate was measured at $267 \mathrm{~nm}$. Quantitation of dUrd was based on external standards. Identification of peaks was based upon retention time and was always confirmed by treatment of a second aliquot of each sample with a large excess of purified TP (Sigma-Aldrich, Milwaukee, Wisconsin, USA) to eliminate dUrd.

DNA analyses. Total DNA from peripheral blood leukocytes, cultured skin fibroblasts, and autopsy tissues was extracted by standard procedures. A series of PCR-amplified fragments of the DNA encompassing first the 22 tRNA genes in mtDNA and then the entire mtDNA was sequenced directly in an ABI Prism 310 Genetic Analyzer using Big Dye Terminator Cycle Sequencing Reaction Kits (Perkin-Elmer Applied Biosystems, Foster City, California, USA) using standard procedures (12). Restriction fragment length polymorphism (RFLP) analyses were performed on DNA from peripheral blood leukocytes, cultured skin fibroblasts, and autopsy tissues.
Cloning the PCR-amplified hypervariable segments. The existence of hypervariable segments (HVSs) in the noncoding human mtDNA control region has been well documented in numerous analyses of human mtDNA variation, and HVS 1 and HVS 2 are thought to be mutational hot spots (13-15). To screen HVS 1 and HVS 2, fragments spanning nucleotides 15,975 to 635 (16) were amplified by PCR from purified DNA samples using a forward primer (nucleotides 15,975-15,994), a backward primer (nucleotides 635-616), and Platinum Taq DNA Polymerase High Fidelity (Invitrogen Corp.). The PCR cycle conditions were: one cycle at $94^{\circ} \mathrm{C}$ for 2 minutes; 30 cycles at $94^{\circ} \mathrm{C}$ for 1 minute, $55^{\circ} \mathrm{C}$ for 1 minute, and $72^{\circ} \mathrm{C}$ for 1 minute; and an extension cycle at $72^{\circ} \mathrm{C}$ for $7 \mathrm{~min}$ utes. The PCR product was isolated by electrophoresis in a $2 \%$ agarose gel. The 1,229-bp band was extracted from the gel using a QIAquick gel extraction kit (QIAGEN Inc., Valencia, California, USA). The PCR product was ligated into PCRII-TOPO vector and subcloned using a TOPO TA Cloning Kit (Invitrogen Corp.) in LB plates containing ampicillin (Invitrogen Corp.) and 5-bromo-4-chloro-3-indolyl$\beta$-D-galactoside. Individual white colonies were cultured independently in TB medium with ampicillin. Approximately 100 cloned plasmids were purified using the High Pure Plasmid Isolation Kit (Roche Diagnostics Corp., Indianapolis, Indiana, USA) and were sequenced directly in an ABI Prism 3700 Genetic Analyzer (Perkin-Elmer Applied Biosystems) following standard procedures (12).

RFLP analyses. RFLP analyses were performed on DNA from peripheral blood leukocytes, cultured skin fibroblasts, and autopsy tissues. For the T5814C mtDNA mutation ( $A 5814 \mathrm{G}$ in the coding heavy strand), a 98-bp mtDNA fragment spanning nucleotides 5,752 to 5,849 was amplified from total DNA samples using a forward mismatch primer (5'-AGGCGGGAGAAGCCCCGGCAGGTTTGAAGCTGCTTCTTAGG-3') (nucleotides 5,752-5,791, mismatch nucleotide underlined) and a backward mismatch primer (5'-CAGGGGTTAGGCCTCTTTTTACCAGCTCCGAGTCG-3') (nucleotides 5,849-5,815) (16). The mutant mtDNA introduces a TaqI restriction site detectable by RFLP analysis. This enzyme cuts the wildtype 98-bp fragment, producing two fragments (64 bp and $34 \mathrm{bp}$ ); however, the T-to-C mutation at nucleotide 5,814 eliminates the restriction site. Digested PCR products were electrophoresed in $12 \%$ nondenaturing acrylamide gels, which were analyzed in a Molecular Imager System GS-363 (Bio-Rad Laboratories Inc., Hercules, California, USA) to quantitate mutation percentages. We also identified the other seven T-to-C point mutations (nucleotides 616, 3,386, 4,370, 10,221, $12,310,15,956$, and 16,172 ) and one T-to-A point mutation at nucleotide 5,628 using RFLP analyses. For the T616C mutation, a 60-bp mtDNA fragment spanning nucleotides 595 to 654 was amplified from total DNA samples using a forward primer (5'-CTCAAAGCAATACACTGAAA) (nucleotides 595-614) and a backward 
Table 1

Mitochondrial enzyme activities in cultured skin fibroblasts from MNGIE patients and controls

\begin{tabular}{lccc}
\hline Fibroblasts & COX $^{\mathrm{A}}$ & $\mathrm{CS}^{\mathrm{B}}$ & $\mathrm{COX} / \mathrm{CS}^{\mathrm{C}}$ \\
Control 1 & 23.8 & 23.4 & 1.02 \\
Control 2 & 19.0 & 24.0 & 0.79 \\
Control 3 & 39.2 & 58.2 & 0.67 \\
MN1-4 & 20.9 & 31.8 & 0.66 \\
MN3-1 & 3.2 & 21.3 & 0.15 \\
MN4-1 & 8.0 & 77.7 & 0.10 \\
MN4-3 & 14.8 & 49.6 & 0.30 \\
MN7-1 & 2.5 & 37.8 & 0.07 \\
MN7-2 & 8.0 & 46.1 & 0.17
\end{tabular}

AAmount of cytochrome $c(\mathrm{nmol})$ oxidized $/ \mathrm{min} / \mathrm{mg}$ protein; COX activities in MNGIE patients' fibroblasts were significantly less than in control cells $(P=0.04)$; Bamount of oxaloacetate ( $\mathrm{nmol}$ ) transformed/min/mg protein; and ${ }^{\mathrm{C}} \mathrm{COX}$ activities normalized to CS were also significantly lower in MNGIE patients' fibroblasts compared to control cells $(P=0.02)$. CS, citrate synthase.

mismatch primer (5'-ACCTATTTGTTTATGGGGTGATGTGAGCCCGTCGAGA-3') (nucleotides 654-618). The mutant mtDNA introduces a $B s m B I$ restriction site. For the T3386C mutation, a 78-bp mtDNA fragment spanning nucleotides 3,350 to 3,427 was amplified from total DNA samples using a forward primer (5'-TCGCAATGGCATTCCTAATGCTTACCGAACG-3') (nucleotides $3,350-3,380)$ and a backward mismatch primer (5'CTACCACGTTGGGGCCTTTGCGTAGTTGTATATAGCCTC G-3') (nucleotides 3,427-3,388). The mutant mtDNA introduces an AvaI restriction site. For the T4370C mutation, a 105-bp mtDNA fragment spanning nucleotides 4,308 to 4,412 was amplified from total DNA samples using a forward primer (5'-GGAGCTTAAACССССТТАTT) (nucleotides 4,308-4,327) and a backward mismatch primer (5'-CTGACCTTACTTTAGGATGGGGTGTGATAGGTGGCACGAA-3') (nucleotides 4,412-4,373). The mutant mtDNA introduces an EarI restriction site. For the $T 10221 \mathrm{C}$ mutation, a 130-bp mtDNA fragment spanning nucleotides 10,171 to 10,300 was amplified from total DNA samples using a forward primer (5'-AGTGCGGCTTCGACCCTATA-3') (nucleotides 10,171-10,190) and a backward primer $\left(5^{\prime}\right.$ CGTTTGTAGGGCTCATGGTAG-3') (nucleotides 10,30010,281). The mutant mtDNA introduces an EarI restriction site. For the T12310C mutation, a 70-bp mtDNA fragment spanning nucleotides 12,277 to 12,346 was amplified from total DNA samples using a forward primer (5'-ATAACAGCTATCCATTGGTCTTAGG) (nucleotides 12,277-12,301) and a backward mismatch primer (5'-GCATGGTTATTACTTTTATTTGGAGTTGCACCAGTA$3^{\prime}$ ) (nucleotides 12,346-12,311). The mutant mtDNA introduces an ScaI restriction site. For the T15956C mutation, a 78-bp mtDNA fragment spanning nucleotides 15,919 to 15,996 was amplified from total DNA samples using a forward mismatch primer $\left(5^{\prime}\right.$ TGTAAAC GGGAGATGAAAACCTTTTTCCAAGG) (nucleotides 15,919-15,950) and a backward mismatch primer (5'-GCTTTGGGTGCTAATGGTGGAGTTAAAGAСТTTTTCTCCG-3') (nucleotides 15,996-15,957). The mutant mtDNA introduces an $M s p I$ restriction site. To detect the T16172C transition, a 69-bp mtDNA fragment spanning nucleotides 16,140 to 16,208 was amplified from total DNA samples using a forward primer ( $5^{\prime}$ - TACTTGACCACCTGTAGTACATAAAAACCCA) (nucleotides 16,140-16,170) and a backward mismatch primer ( 5 '-CTTGCTTGTAAGCATGGGGAGGGGGTTTTGATCCGG-3') (nucleotides 16,208 to 16,173 , mismatch nucleotide underlined) (16). The mutant mtDNA introduces an NciI restriction site. For the T5628A mutation, a 120-bp mtDNA fragment spanning nucleotides 5,549 to 5,668 was amplified from total DNA samples using a forward primer (5'-GCCCTCAGTAAGTTGCAATA) (nucleotides 5,549 to 5,568 ) and a backward mismatch primer (5'-CCATTGGTCTAGTAAGGGCTTAGCTTAATTAAAGTG $\underline{C}$ C $\underline{A}-3^{\prime}$ ) (nucleotides 5,668-5,629). The mutant mtDNA introduces a $B s r I$ restriction site.

Statistical methods. We used the Mann-Whitney $U$ nonparametric test to assess differences in biochemical activities of skin fibroblasts because of the low number of samples $(n<10)$.

\section{Table 2}

mtDNA point mutations in fibroblasts of MNGIE patients identified by sequencing

\begin{tabular}{|c|c|c|c|c|}
\hline \multirow[b]{2}{*}{$\mathrm{T}$ position } & \multicolumn{2}{|c|}{ Skin fibroblasts } & \multirow[b]{2}{*}{ Region } & \multirow[b]{2}{*}{ Skeletal mus } \\
\hline & Sequence & Mutation & & \\
\hline \multicolumn{5}{|l|}{ MN4-2 } \\
\hline $291(H)$ & AAAT & $\mathrm{T} \rightarrow \mathrm{A}$ & D-loop & $\mathrm{n}$ \\
\hline $2,805(\mathrm{H})$ & AAAT & $\mathrm{T} \rightarrow \mathrm{A}$ & $16 \mathrm{~S}$ & $\mathrm{n}$ \\
\hline $8,367(\mathrm{~L})$ & AAAT & $\mathrm{T} \rightarrow \mathrm{C}$ & ATPase 8 & $\mathrm{n}$ \\
\hline $14,880(\mathrm{~L})$ & AAAT & $\mathrm{T} \rightarrow \mathrm{A}$ & CytB & $\mathrm{n}$ \\
\hline $3,653(\mathrm{~L})$ & AAT & $\mathrm{T} \rightarrow \mathrm{C}$ & ND1 & $\mathrm{n}$ \\
\hline $4,028(\mathrm{~L})$ & AAT & $\mathrm{T} \rightarrow \mathrm{C}$ & ND1 & $\mathrm{n}$ \\
\hline $8,108(\mathrm{H})$ & AAT & $\mathrm{T} \rightarrow \mathrm{C}$ & $\operatorname{cox} 2$ & $\mathrm{n}$ \\
\hline $14,166(\mathrm{~L})$ & AAT & $\mathrm{T} \rightarrow \mathrm{C}$ & ND6 & $\mathrm{n}$ \\
\hline $15,831(\mathrm{~L})$ & AAT & $\mathrm{T} \rightarrow \mathrm{C}$ & CytB & $\mathrm{n}$ \\
\hline $9,758(L)$ & $C T$ & $\mathrm{~T} \rightarrow \mathrm{C}$ & $\operatorname{cox} 3$ & $\mathrm{n}$ \\
\hline \multicolumn{5}{|l|}{ MN7-2 } \\
\hline $2,233(\mathrm{~L})$ & AAAAAAT & $\mathrm{T} \rightarrow \mathrm{C}$ & $16 \mathrm{~S}$ & $\mathrm{n}$ \\
\hline $7,440(\mathrm{~L})$ & AAAAT & $\mathrm{T} \rightarrow \mathrm{C}$ & $\operatorname{cox} 1$ & $n$ \\
\hline $13,879(\mathrm{~L})$ & AAAAT & $\mathrm{T} \rightarrow \mathrm{C}$ & ND5 & $\mathrm{n}$ \\
\hline $291(\mathrm{H})$ & AAAT & $\mathrm{T} \rightarrow \mathrm{A}$ & D-loop & $\mathrm{n}$ \\
\hline $2,805(\mathrm{H})$ & AAAT & $\mathrm{T} \rightarrow \mathrm{A}$ & $16 \mathrm{~S}$ & $\mathrm{n}$ \\
\hline $5,517(\mathrm{~L})$ & AAAT & $\mathrm{T} \rightarrow \mathrm{C}$ & tRNA $A^{T r y}$ & $n$ \\
\hline $5,628(\mathrm{~L})$ & AAAT & $\mathrm{T} \rightarrow \mathrm{C}$ & tRNA $\mathrm{Ala}^{\mathrm{Ala}}$ & $n$ \\
\hline $10,432(\mathrm{H})$ & AAAT & $\mathrm{T} \rightarrow \mathrm{C}$ & tRNA Arg & $\mathrm{n}$ \\
\hline $8,108(\mathrm{H})$ & AAT & $\mathrm{T} \rightarrow \mathrm{C}$ & $\operatorname{cox} 2$ & $\mathrm{n}$ \\
\hline $9,110(\mathrm{~L})$ & AAT & $\mathrm{T} \rightarrow \mathrm{C}$ & ATPase 6 & $\mathrm{n}$ \\
\hline $1,040(\mathrm{~L})$ & AT & $\mathrm{T} \rightarrow \mathrm{C}$ & $12 S$ & $\mathrm{n}$ \\
\hline $11,781(\mathrm{~L})$ & AT & $\mathrm{T} \rightarrow \mathrm{C}$ & ND4 & $\mathrm{n}$ \\
\hline $4,160(\mathrm{~L})$ & $\mathrm{AACT}$ & $\mathrm{T} \rightarrow \mathrm{A}$ & ND1 & $n$ \\
\hline $7,055(\mathrm{H})$ & $C T$ & $\mathrm{~T} \rightarrow \mathrm{C}$ & $\operatorname{cox} 1$ & $\mathrm{n}$ \\
\hline $11,324(\mathrm{~L})$ & AAACT & $\mathrm{T} \rightarrow \mathrm{C}$ & ND4 & $\mathrm{n}$ \\
\hline
\end{tabular}

MNGIE patient DNA from passage 3 cultured skin fibroblasts and skeletal muscle. Numbers indicate nucleotide position in mtDNA (16). L, mtDNA light strand; $\mathrm{H}$, heavy strand; 16S, 16S rRNA; 12S, 12S rRNA; CytB, cytochrome $b$; $\mathrm{ND}$, nicotinamide adenine dinucleotide dehydrogenase; $\mathrm{n}$, negative. 
Table 3

mtDNA point mutations in MNGIE patients screened by RFLP analysis

\begin{tabular}{|c|c|c|c|c|c|c|c|c|c|c|c|}
\hline & & & & & & Light strand & & & & & Heavy strand \\
\hline & & Sequence & AAT & AA & AT & & $\mathrm{AA} A$ & ATT & & AAAAAT & AAAAT \\
\hline & & $\mathrm{T}$ position & 16172 & 5628 & 15956 & 616 & 4370 & 5814 & 10221 & 3386 & 12310 \\
\hline & & Mutation & $\mathrm{T} \rightarrow \mathrm{C}$ & $\mathrm{T} \rightarrow \mathrm{A}$ & $\mathrm{T} \rightarrow \mathrm{C}$ & $\mathrm{T} \rightarrow \mathrm{C}$ & $\mathrm{T} \rightarrow \mathrm{C}$ & $\mathrm{T} \rightarrow \mathrm{C}$ & $\mathrm{T} \rightarrow \mathrm{C}$ & $\mathrm{T} \rightarrow \mathrm{C}$ & $\mathrm{T} \rightarrow \mathrm{C}$ \\
\hline & & AA Change & & & & & & & Phe $\rightarrow$ Leu & $\mathrm{Ile} \rightarrow$ Thr & \\
\hline Patient & Tissue & Region & D-loop & tRNA ${ }^{A l a}$ & tRNA ${ }^{\text {Pro }}$ & tRNA $A^{\text {Phe }}$ & tRNA ${ }^{\mathrm{Gln}}$ & tRNA ${ }^{\text {Cys }}$ & ND3 & ND1 & tRNA $A^{\text {Leu(CUN) }}$ \\
\hline MN1-1 & Leukocytes & & 19 & $n$ & $<2$ & $\mathrm{n}$ & $\mathrm{n}$ & 11 & 2 & 2 & $\mathrm{n}$ \\
\hline MN1-2 & Leukocytes & & 41 & $<2$ & $<2$ & $\mathrm{n}$ & $\mathrm{n}$ & 17 & 2 & 3 & $\mathrm{n}$ \\
\hline MN1-3 & Leukocytes & & 16 & $\mathrm{n}$ & $<2$ & $\mathrm{n}$ & $\mathrm{n}$ & 10 & $<2$ & 2 & $\mathrm{n}$ \\
\hline MN1-4 & Skin fibroblasts & & 38 & $<2$ & 14 & $\mathrm{n}$ & $<2$ & 40 & 4 & 9 & 26 \\
\hline & Leukocytes & & 22 & $<2$ & $<2$ & $n$ & $\mathrm{n}$ & 6 & 2 & 2 & $\mathrm{n}$ \\
\hline MN2-1 & Cardiac muscle & & $\mathrm{n}$ & $<2$ & $\mathrm{n}$ & $\mathrm{n}$ & $\mathrm{n}$ & 2 & - & $\mathrm{n}$ & $\mathrm{n}$ \\
\hline & Small intestine & & 31 & $<2$ & $<2$ & $\mathrm{n}$ & $\mathrm{n}$ & 10 & - & $\mathrm{n}$ & $\mathrm{n}$ \\
\hline & Leukocytes & & 14 & $<2$ & $<2$ & $\mathrm{n}$ & $\mathrm{n}$ & 4 & - & $\mathrm{n}$ & $\mathrm{n}$ \\
\hline MN2-2 & Leukocytes & & 13 & $\mathrm{n}$ & $<2$ & $\mathrm{n}$ & $\mathrm{n}$ & 9 & - & $\mathrm{n}$ & $n$ \\
\hline MN3-1 & Small intestine & & 35 & $\mathrm{n}$ & $<2$ & $\mathrm{n}$ & $\mathrm{n}$ & 19 & 5 & $\mathrm{n}$ & $\mathrm{n}$ \\
\hline & Skin fibroblasts & & $\mathrm{n}$ & $n$ & 9 & $\mathrm{n}$ & $n$ & 76 & 2 & $n$ & $n$ \\
\hline & Leukocytes & & 15 & $\mathrm{n}$ & $<2$ & $\mathrm{n}$ & $\mathrm{n}$ & 7 & 2 & $\mathrm{n}$ & $\mathrm{n}$ \\
\hline MN4-1 & Leukocytes & & $\mathrm{n}$ & $\mathrm{n}$ & $\mathrm{n}$ & $\mathrm{n}$ & $\mathrm{n}$ & 4 & $\mathrm{n}$ & $\mathrm{n}$ & $\mathrm{n}$ \\
\hline MN4-2 & Small intestine & & 14 & 2 & 15 & $\mathrm{n}$ & $\mathrm{n}$ & 8 & 2 & 2 & $\mathrm{n}$ \\
\hline & Skin fibroblasts & & 39 & 2 & 5 & $n$ & $n$ & 7 & 10 & 3 & $n$ \\
\hline & Leukocytes & & 6 & $n$ & $\mathrm{n}$ & $n$ & $n$ & 3 & $\mathrm{n}$ & $\mathrm{n}$ & $n$ \\
\hline MN4-3 & Skin fibroblasts & & 69 & 34 & 28 & $\mathrm{n}$ & 41 & 56 & $<2$ & 2 & $\mathrm{n}$ \\
\hline & Leukocytes & & $\mathrm{n}$ & $\mathrm{n}$ & $\mathrm{n}$ & $\mathrm{n}$ & $\mathrm{n}$ & 3 & $\mathrm{n}$ & $\mathrm{n}$ & $n$ \\
\hline MN6-1 & Leukocytes & & 15 & $n$ & $<2$ & $\mathrm{n}$ & $\mathrm{n}$ & 7 & - & $\mathrm{n}$ & $\mathrm{n}$ \\
\hline MN7-1 & Skin fibroblasts & & 28 & $n$ & $<2$ & $\mathrm{n}$ & 14 & 81 & 5 & 4 & $\mathrm{n}$ \\
\hline & Leukocytes & & 11 & $n$ & $<2$ & $\mathrm{n}$ & $\mathrm{n}$ & 5 & $<2$ & n & n \\
\hline MN7-2 & Skin fibroblasts & & 7 & 5 & 2 & 4 & 29 & 6 & 3 & 3 & $\mathrm{n}$ \\
\hline & Leukocytes & & 19 & $\mathrm{n}$ & $<2$ & $\mathrm{n}$ & $\mathrm{n}$ & 6 & $<2$ & $\mathrm{n}$ & $n$ \\
\hline
\end{tabular}

Mutation levels expressed as percentages. DNA from peripheral blood leukocytes, passage 4 (MN4-2, MN4-3, MN7-1, and MN7-2) or passage 5 (MN1-4 and MN3-1) cultured skin fibroblasts, cardiac muscle, and small intestine. $n$, negative; -, not determined.

\section{Results}

COX in skin fibroblasts. COX activity was decreased in the skin fibroblast lines of MNGIE patients compared with control cells $(P=0.04)$ (Table 1$)$. The skin fibroblasts with normal COX activity came from a mildly affected individual (MN1-4). In contrast to COX, activity of citrate synthase (a mitochondrial matrix enzyme and a measure of mitochondrial mass) was similar in the two groups (Table 1). When COX activity was normalized to citrate synthase, the defects of COX activity in patient skin fibroblasts were more evident $(P=0.02)$ (Table 1$)$.

Levels of dUrd in MNGIE patients. Plasma levels of dUrd were undetectable in the controls $(n=20)$ and heterozygous TP mutation carriers $(n=14)$ (i.e., concentrations were below $0.05 \mu \mathrm{M}$ ) but were markedly elevated in all patients analyzed $(14.2 \mu \mathrm{M} \pm 4.4 \mu \mathrm{M}$, mean \pm SD; range 5.5-24.4; $n=25$ ).

mtDNA point mutations in MNGIE patients. Based upon our observation that TP-deficient skin fibroblasts have decreased COX activity (Table 1) but do not show depletion or multiple deletions of mtDNA by Southern blot analyses (data not shown), we suspected that mtDNA point mutations might be present in cells from MNGIE patients. We therefore sequenced the $22 \mathrm{mtDNA}$-encoded tRNA genes from cultured skin fibroblast DNA from six MNGIE patients (MN1-4, MN3-1, MN4-2, MN4-3, MN7-1, and MN7-2). We identified three T-to-C transitions: at nucleotide 4,370 in $\mathrm{tRNA}^{\mathrm{Gln}}$, at nucleotide 5,814 in $\mathrm{tRNA}^{\mathrm{Cys}}$, and at nucleotide 15,956 in tRNA ${ }^{\text {Pro }}$ (16). Interestingly, all three mutations were preceded by three A residues (i.e., 5'-AAAT to 5'-AAAC).

In addition, we sequenced the entire mtDNA of skeletal muscle and cultured skin fibroblasts from two MNGIE patients (MN4-2 and MN7-2). To identify maternally inherited polymorphisms, we also sequenced peripheral blood leukocyte mtDNA from the mothers of both patients. We did not find any mutations in the skeletal muscle mtDNA of the patients. In contrast, we identified 22 additional mtDNA point mutations in cultured skin fibroblasts from the two MNGIE patients (Table 2). Interestingly, 18 of these point mutations (82\%) (nucleotides 1,040, 2,233, 3,653, 4,028, 5,517, 5,628, 7,055, 7,440, 8,108, 8,367, 9,110, 9,758, 10,432, 11,324, 11,781, 13,879, $14,166$, and 15,831$)$ were T-to-C transitions. Fifteen of these $18 \mathrm{~T}$-to- $\mathrm{C}$ transitions $(83 \%)$ were preceded by at least one A residue (Table 2), and seven transitions (39\%) were preceded by at least three A residues.

To quantitate the levels of the mtDNA mutations and to identify additional 5 '-AAAT to 5 '-AAAC transitions, RFLP analyses were performed on DNA extracted from six cultured skin fibroblast lines, 13 
peripheral blood leukocyte samples, and four autopsy tissue samples from MNGIE patients. Of the 203 5 -AAAT sites in mtDNA, eight that were amenable to RFLP analysis were screened along with one 5 '-AAT site. We found six additional heteroplasmic mutations in skin fibroblast mtDNA: four were 5 -AAAT to 5 -AAAC mutations located at nucleotide 616 in tRNA $^{\text {Phe }}$, at nucleotide 3,386 in subunit 1 of nicotinamide adenine dinucleotide dehydrogenase-1, at nucleotide 10,221 in nicotinamide adenine dinucleotide dehydrogenase- 3 , and at nucleotide 12,310 in tRNA ${ }^{\mathrm{Leu}(\mathrm{CUN})}$. The fifth mutation was a $5^{\prime}$-AAT to $5^{\prime}$-AAC transition at nucleotide 16,172 in the displacement loop (D-loop). The sixth mutation we identified by RFLP analysis was a heteroplasmic 5'AAAT to $5^{\prime}$-AAAA mutation at nucleotide 5,628 in tRNA ${ }^{\text {Ala }}$ of skin fibroblast, peripheral blood leukocyte, and tissue mtDNA from six patients (Table 3 ). T5628C was identified as a pathogenic mutation in a family with maternally inherited progressive external ophthalmoplegia (17).

Overall, of the nine mutations screened by RFLP analyses, seven (nucleotides 3,386, 4,370, 5,628, 5,814, $10,221,15,956$, and 16,172) were identified in at least half of the patients' skin fibroblasts, and three (nucleotides 5,814, 10,221, and 15,956) were present in all six cell lines, with heteroplasmic levels ranging from below $2 \%$ to $81 \%$ (Table 3 ). In addition, five mutations (nucleotides 3,386, 5,814, 10,221, 15,956, and 16,172 ) were detected by RFLP analysis in leukocytes and in autopsy tissues from patients (MN2-1, MN3-1, MN4-2, and MN7-2), with levels of heteroplasmy at or below 63\% (Table 3 and Table 4). Remarkably, all tissue and cell samples from the 13 patients tested contained the nucleotide 5,814 mutation. T5814C was identified as a pathogenic mutation ( $A 5814 \mathrm{G}$ in the coding heavy strand) in patients with mitochondrial encephalopathy $(18,19)$ or mitochondrial myopathy and cardiomyopathy (20).

All of the mutations were absent in 33 control samples from 22 individuals (six cultured skin fibroblast lines, 11 brains, four small intestines, six kidneys, and six livers) and in unaffected carriers of TP mutations (23 peripheral blood leukocyte samples) (data not shown).

To determine whether analogous mutations were present in nuclear DNA from MNGIE patients, we performed RFLP screening analyses of seven genomic $5^{\prime}$ AAAT sites, one in the TP gene itself, three in the $18 \mathrm{~S}$ ribosomal RNA gene (21), and three in the human $\beta$-actin gene (22), using total DNA from six skin fibroblast lines, 13 peripheral blood leukocyte samples, and 32 tissues obtained from 13 MNGIE patients. No mutations were detected (data not shown).

mtDNA point mutations in MNGIE patient HVSs detected by sequencing of PCR clones. To screen for mutations at non-5'-AAAT sites, we sequenced HVS 1 and HVS 2 in the mtDNA D-loop, which are considered to be hot spots for mutations (13-15). We subcloned PCRamplified HVSs of cultured skin fibroblast mtDNA from two patients (MN4-3 and MN7-1) and two controls, and sequenced between 84 and 92 clones of each PCR fragment. We identified four additional $5^{\prime}$-AAAT to $5^{\prime}$-AAAC heteroplasmic mutations at nucleotides 291, 292, 16,043, and 16,352 (Table 5). In addition, we identified four other mutations that were not 5'-AAAT to 5'-AAAC: $5^{\prime}$-GGAGT to $5^{\prime}$-GGAGC at nucleotide $16,247,5^{\prime}$-AAAT to $5^{\prime}$-AAAA at nucleotide 291, 5'-AAATT to 5'-AAAAA at nucleotides 291-290,

\section{Table 4}

mtDNA point mutations in autopsy tissues of MNGIE patients screened by RFLP analysis

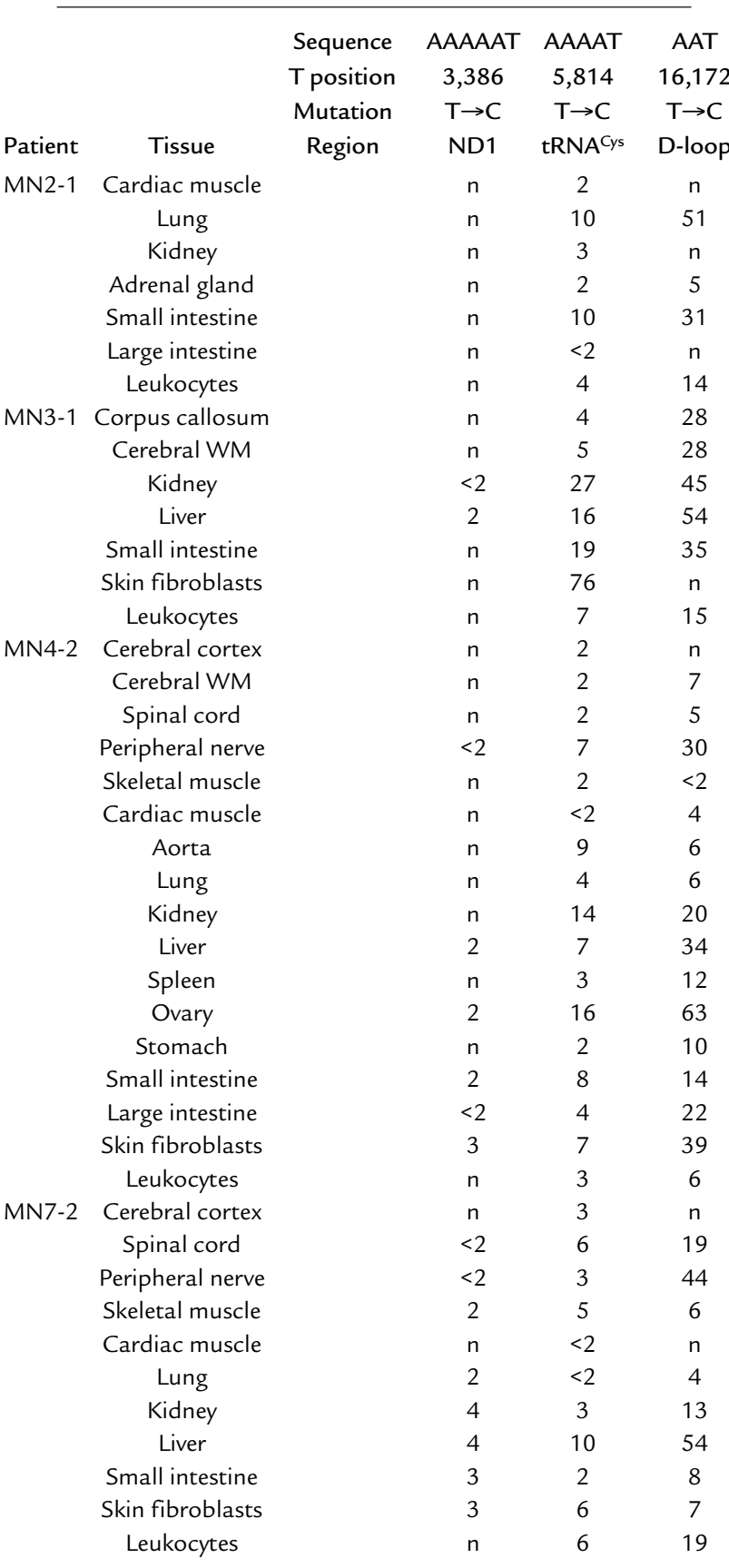

Mutation levels expressed as percentages. WM, occipital white matter. Skin fibroblasts were passage 4 (MN4-2 and MN7-2) or passage 5 (MN3-1) cultured skin fibroblasts. Leukocytes, peripheral blood leukocytes; n, negative. 


\begin{tabular}{|c|c|c|c|c|c|c|c|c|c|c|c|}
\hline & \multirow[b]{2}{*}{$\begin{array}{l}\text { Number of } \\
\text { clones }\end{array}$} & \multirow[b]{2}{*}{$\begin{array}{c}\text { Sequence } \\
\text { T position } \\
\text { mtDNA mutation }\end{array}$} & \multicolumn{3}{|c|}{ Light strand } & \multicolumn{6}{|c|}{ Heavy strand } \\
\hline & & & $\begin{array}{c}\text { AAAAAAT } \\
292 \\
T \rightarrow C\end{array}$ & $\begin{array}{c}\text { AAAT } \\
16,352 \\
T \rightarrow C\end{array}$ & $\begin{array}{c}\text { AAT } \\
16,172 \\
\mathrm{~T} \rightarrow \mathrm{C}\end{array}$ & $\begin{array}{c}\text { AAAT } \\
16,043 \\
\mathrm{~T} \rightarrow \mathrm{C}\end{array}$ & $\begin{array}{l}\text { AAAT } \\
291 \\
T \rightarrow \text { del }\end{array}$ & $\begin{array}{l}\text { AAAT } \\
291 \\
\mathrm{~T} \rightarrow \mathrm{C}\end{array}$ & $\begin{array}{l}\text { AAAT } \\
291 \\
\mathrm{~T} \rightarrow \mathrm{A}\end{array}$ & $\begin{array}{l}\text { AAATT } \\
291-290 \\
\text { TT } \rightarrow \text { AA }\end{array}$ & $\begin{array}{l}\text { GGAGT } \\
16,247 \\
T \rightarrow C\end{array}$ \\
\hline MN4-3 & $84-90$ & & 0 & 4 & 28 & 26 & 5 & 0 & 77 & 2 & 0 \\
\hline MN7-1 & 92 & & 17 & 2 & 11 & 0 & 0 & 3 & 62 & 0 & 18 \\
\hline
\end{tabular}

Fibroblasts were screened for mtDNA mutations by sequencing PCR clones. del, deleted.

and deletion of a $\mathrm{T}$ at $5^{\prime}$-AAATTTTTT (Table 5). Four 5 '-AAAT sites (at nucleotides 16,094-16,091, $16,137-16,140,16,325-16,322$, and 159-156) in HVS 1 and HVS 2 did not show mutations. DNA from two control skin fibroblast lines did not show any mutations in the D-loop.

\section{Discussion}

In MNGIE, mutations in TP cause elevated levels of dThd in the blood of patients (7). We have hypothesized that in this disease, abnormal nucleoside metabolism leads to alterations of mitochondrial deoxynucleoside $5^{\prime}$-triphosphate (dNTP) pools that in turn generate mtDNA abnormalities such as depletion and multiple deletions $(6,7)$. The alterations of the dThd pathway seem to affect dNTP pools more severely in mitochondria than in nuclei. This selective effect on mtDNA may be explained by three factors. First, mitochondrial dNTP pools are physically separate and are regulated independently (23-25). Second, mitochondrial nucleotide pools are probably more vulnerable to the toxic effects of excessive dThd than are nuclear nucleotide pools because mtDNA is more dependent on dThd salvage than is nuclear DNA, which relies upon the de novo synthesis of dThd $(24,26)$. Third, human mitochondria may lack an effective mismatch repair system (27). Although mtDNA is clearly affected in MNGIE, we cannot exclude the possibility that nuclear DNA is also affected.

In this report, we have identified $36 \mathrm{mtDNA}$ point mutations, a TT-to-AA substitution, and a single nucleotide deletion in cells from MNGIE patients (Table 6). This is the first report of multiple mtDNA point mutations in an autosomal mitochondrial disease. Several features of these mutations are noteworthy. First, point mutations of mtDNA were detected in all MNGIE patients examined (Table 3), and every tissue had at least one mtDNA point mutation (Table 4). Second, we did not find these mutations in any unaffected carriers of TP mutations, implying that the mtDNA alterations were somatic point mutations rather than being maternally inherited. Third, the vast majority of the point mutations identified by sequencing ( 18 of the 22 , or $82 \%$ ) were T-to-C transitions. Fourth, 18 of 22 (82\%) of the mtDNA point mutations identified by sequencing were preceded by at least one A residue, and 10 of 22
(45\%) were preceded by $5^{\prime}$-AAA sequences (Table 2 ). The T-to-C mutations occurred more frequently at $5^{\prime}$-AAAT ( 4 of 139 , or $2.9 \%$ ) and $5^{\prime}$-AAT sites ( 6 of 506, or $1.2 \%)$ than at $5^{\prime}$-AT ( 2 of 1,897 , or $\left.0.1 \%\right)$ sites.

It is uncertain how mtDNA point mutations are produced in MNGIE, but based on the sequence specificity of the alterations, we propose two mechanisms: a "next-nucleotide effect" leading to a high level of direct misincorporation of the incorrect nucleotide (28), and "dislocation mutagenesis" (29) (Figure 1). It has been shown that direct misincorporation of a deoxyguanosine monophosphate (dGMP) opposite a template thymine residue (T.dGMP) is a common event $(30,31)$. After the misincorporation, high concentrations of deoxythymidine triphosphate (dTTP) in the mitochondria of MNGIE cells may accelerate polymerase activity across the $5^{\prime}$-AA template region and thereby compromise the mtDNA polymerase $\gamma(\mathrm{pol} \gamma)$ exonuclease removal of the mismatched T.dGMP nucleotide (Figure 1a). This nextnucleotide effect has been observed in vitro with bacterial and mammalian polymerase with exonuclease activities, including mtDNA pol $\gamma(28,32,33)$. In addition, mtDNA pol $\gamma$ exonuclease activity can be inhibited by elevated levels of deoxythymidine monophosphate (dTMP) derived from the increased dThd $(33,34)$. Therefore, in MNGIE cells, both elevated ATMP and ATTP derived from the increased dThd may contribute to the inhibition of mtDNA pol $\gamma$ exonuclease activity. The T.G misincorporation will result in a T-to-C mutation following a subsequent round of mtDNA replication.

Increased reactive oxygen species produced by dysfunctional mitochondrial respiratory chain enzymes may contribute to the accumulation of T-to-C transitions. Specifically, oxidation of guanine residues to 8-oxoguanine is mutagenic, as it induces mispairing with As and causes G-to-T transversions (C-to-A on the complementary strand) (35); however, the vast majority of the mutations we observed were T-to-C transitions (A-to-G on the complementary strand). Furthermore, in the early stages of MNGIE, imbalances of nucleosides (and presumably nucleotides) must precede the mtDNA alterations and respiratory chain defects. Consequently, reactive oxygen species are unlikely to play a primary role in the generation of the mtDNA point mutations in MNGIE. 
Table 6

Sequences of mtDNA mutations in MNGIE patients

T-to- $C$ sites

$292(\mathrm{~L})$

2,233 (L)

$3,386(\mathrm{~L})$

$616(\mathrm{~L})$

$4,370(\mathrm{~L})$

5,814 (L)

7,440 (L)

10,221 (L)

$12,310(\mathrm{H})$

13,879 (L)

$291(\mathrm{H})$

$5,517(\mathrm{~L})$

$5,628(\mathrm{~L})$

$8,367(\mathrm{~L})$

$10,432(\mathrm{H})$

$15,956(\mathrm{~L})$

$16,043(\mathrm{H})$

$16,352(\mathrm{~L})$

$3,653(\mathrm{~L})$

$4,028(\mathrm{~L})$

$8,108(\mathrm{H})$

$9,110(\mathrm{~L})$

$14,166(\mathrm{~L})$

15,831 (L)

16,172 (L)

$1,040(\mathrm{~L})$

$11,781(\mathrm{~L})$

$16,247(\mathrm{H})$

$7,055(\mathrm{H})$

9,758 (L)

$11,324(\mathrm{~L})$

T-to-A sites

$291(\mathrm{H})$

$2,805(\mathrm{H})$

$5,628(\mathrm{~L})$

14,880 (L)

4,160 (L)

TT-to-AA site

291-290 (H)

T-to-del site

$291(\mathrm{H})$

GGGGGGTTTGGTGGAAATTTTTTTTATGATGTC

Numbers indicate nucleotide position in mtDNA (16). Sequences with underlines indicate 5'-AAT sites; letters in bold indicate mutated T in mtDNA.

Elevated dUrd levels may also contribute to the generation of mtDNA point mutations in MNGIE. In fact, patients have higher plasma levels of dUrd than dThd. Like dThd, dUrd can be phosphorylated via the dThd salvage pathway, leading to abnormally high mitochondrial concentrations of deoxyuridine triphosphate (dUTP) that can substitute for dTTP in mtDNA replication (36). Consequently, the nextnucleotide effect could be amplified by the presence of elevated levels of dUTP in addition to the increased dTTP.
It is likely that additional factors may render specific 5'-AAT sites (e.g., T5814C) more vulnerable to point mutations. Nevertheless, 39 5'-AAT sites in the D-loop did not reveal point mutations, indicating that not all 5 -AAT sites are susceptible to substitutions.

In contrast to the misincorporation mechanism for $5^{\prime}$-AAT to $5^{\prime}$-AAC transitions, a dislocation mutagenesis model could account for the T-to-A, TT-to-AA, and $\mathrm{T}$-deletion mutations observed at nucleotides 291-290 (Figure 1b) (29). According to this proposed mechanism, mutations are produced by a transient single-nucleotide misalignment in the template DNA. For example, after incorporation of deoxyadenosine monophosphate (dAMP) opposite the template's penultimate base in a stretch of six $\mathrm{T}$ residues (nucleotides 285-291), a $\mathrm{T}$ residue within the homopolymeric region loops out and leads to the incorporation of dTMP opposite the adjacent A residue (Figure 1b, step immediately below "Slippage"). If subsequent nucleotides are incorporated before the extrahelical $\mathrm{T}$ realigns, a single $\mathrm{T}$ deletion will result. In support of this mechanism, an in vitro assay demonstrated that pol $\gamma$ frequently generated single $\mathrm{T}$ deletions in homopolymeric $\mathrm{T}$ runs $(29,30)$. Alternatively, if realignment of the extrahelical $\mathrm{T}$ residue occurs before the next nucleotide is incorporated (Figure 1b, under "Realignment"), then the 3 'end $T$ of the daughter strand will align inappropriately with a template $T$ (29). Subsequently, the next-nucleotide effect may accelerate mtDNA pol $\gamma$ polymerization and inhibit exonuclease repair of the mismatched nucleotide. In this situation, the T.T mispair is formed - not by direct misincorporation, but by the transient misalignment - and leads to a Tto-A transversion as observed in the in vitro mtDNA pol $\gamma$ medication assay (29) (Figure 1). Of note, an analysis of published HVS 1 and HVS 2 polymorphisms in various human populations suggested that dislocation mutagenesis contributes to the generation of base substitutions, particularly in monotonous runs of nucleotides (15). In contrast to our observations in MNGIE patients, previous studies of mutations produced by mtDNA pol $\gamma$ in vitro have not revealed enhanced point mutations in short homopolymeric runs; however, these studies have used balanced nucleotide pools (29).

The mtDNA point mutations in MNGIE are likely to contribute to the disease pathogenesis by causing respiratory chain enzyme deficiencies. Many of these mutations are present at levels too low to be of functional significance and most are likely to be neutral polymorphisms. Nevertheless, as previously noted, two of the T-to-C transitions, at nucleotides 5,628 and 5,814, have been described as primary pathogenic mtDNA mutations in patients with mitochondrial encephalomyopathies (17-20) and are likely to contribute to the mitochondrial dysfunction. The levels of heteroplasmy of these mtDNA mutations in the patient's tissues are below the typical $80-90 \%$ 


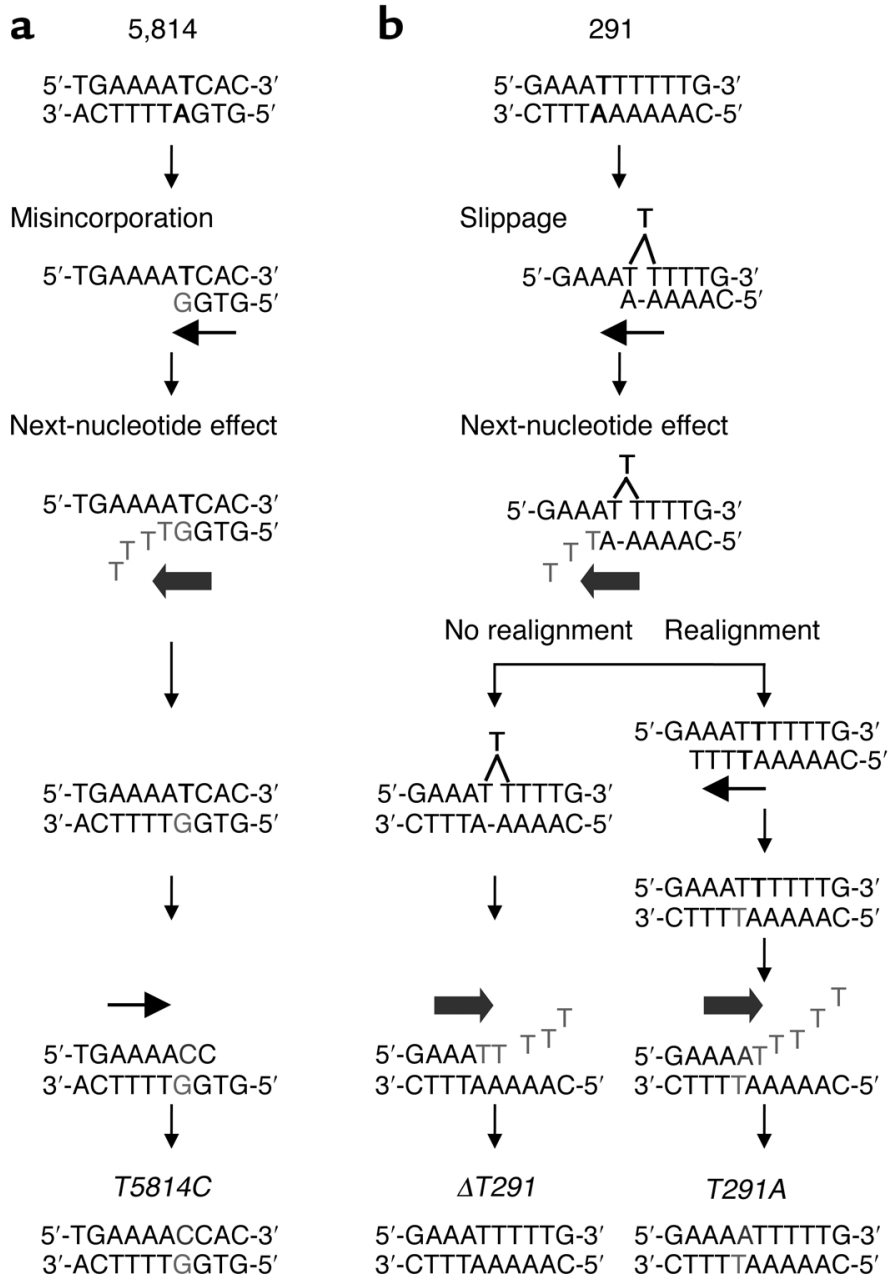

Figure 1

(a) Model of misincorporation and next-nucleotide effect. (b) Model of dislocation mechanism with the next-nucleotide effect.

threshold required to produce a biochemical phenotype. However, focal accumulation of very high levels of individual mtDNA point mutations is likely to be pathogenic in individual cells or segments of skeletal muscle cells. In fact, focal accumulation of pathogenic mtDNA deletions has been identified in COXdeficient skeletal muscle of patients with autosomal dominant progressive external ophthalmoplegia (37) and inclusion body myopathy (38).

The relative pathogenic contributions of the three forms of mtDNA abnormalities in MNGIE- namely depletion, multiple deletions, and point mutations are likely to vary from tissue to tissue. For example, in skin fibroblasts, we have detected only point mutations without depletion or multiple deletions of mtDNA. In contrast, in skeletal muscle, depletion and multiple deletions of mtDNA are probably of greater pathogenic significance than mtDNA point mutations, which were not detected by complete mtDNA sequencing (Table 2 ) and were shown to be present only at low levels $(\leq 2 \%)$ by RFLP analysis
(Table 3 and Table 4). The relatively high levels of mtDNA point mutations in cultured fibroblasts may be due to the fact that the cells primarily use anaerobic glycolysis rather than oxidative phosphorylation to generate ATP. Consequently, in the absence of selective pressure against cells or mitochondria with respiratory chain defects, unbalanced mitochondrial nucleotide pools may generate high levels of mtDNA point mutations.

Sequencing of mtDNA deletion junctions has not revealed a relationship with the $5^{\prime}$-AAT sites $(1,39)$. Nevertheless, the point mutations in the D-loop could alter mtDNA replication, leading to mtDNA depletion. However, it may be difficult to detect such mutations since such replication-incompetent molecules would be present at very low levels. The mtDNA mutations in the D-loop that we have identified probably do not impair replication because they do not affect sequences such as the conserved sequence blocks or termination association sequences that are important for mtDNA replication (40).

It is possible that other conditions that produce imbalances of deoxynucleotide pools with elevation of at least one dNTP species could cause site-specific point mutations in $\mathrm{mtDNA}$ or nuclear DNA. For example, loss-offunction mutations in deoxynucleotidase-2 (dNT-2), which dephosphorylates dTMP and dUTP in mitochondria, may also increase dTTP and dUTP pools in mitochondria, leading to mtDNA alterations similar to those in MNGIE (41). By contrast, loss-of-function mutations in the gene encoding the cytosolic enzyme dNT-1 may produce nuclear DNA mutations (41).

In summary, we have identified 38 distinct mtDNA mutations in tissues, peripheral blood leukocytes, and cultured skin fibroblasts from MNGIE patients harboring loss-of-function TP mutations. Absence of the mutations in unaffected matrilineal relatives of the patients indicates that the mutations arose in somatic tissues. Most of the point mutations were site-specific; 25 of 36 were T-to-C transitions preceded by at least two A residues. Our prior work has demonstrated alterations of dThd and dUrd metabolism in MNGIE patients (7) and has led us to hypothesize that the elevated levels of both nucleosides generate nucleotide pool imbalances in mitochondria, causing the accumulation of point mutations, multiple deletions, and depletion of mtDNA. Measurements of nucleotide pools in mitochondria, nuclei, and cytoplasm will be required to prove this hypothesis, but are technically challenging. Alterations of nucleoside metabolism leading to sequencespecific point mutations may be a significant new disease mechanism in humans. 


\section{Acknowledgments}

The authors thank Eric A. Schon, Thomas A. Kunkel, and Rodney Rothstein for helpful discussions. The authors also acknowledge Michael T. Lin for assistance with DNA sequencing and Saba Tadesse for excellent technical support. Normal human autopsy samples were obtained from the Brain and Tissue Bank, University of Maryland. This work was supported by NIH grant R01 HD-37529 and by a grant from the Muscular Dystrophy Association.

1. Hirano, M., et al. 1994. Mitochondrial neurogastrointestinal encephalomyopathy (MNGIE): clinical, biochemical, and genetic features of an autosomal recessive mitochondrial disorder. Neurology. 44:721-727.

2. Nishino, I., et al. 2000. Mitochondrial neurogastrointestinal encephalomyopathy: an autosomal recessive disorder due to thymidine phosphorylase mutations. Ann. Neurol. 47:792-800.

3. Papadimitriou, A., et al. 1998. Partial depletion and multiple deletions of muscle mtDNA in familial MNGIE syndrome. Neurology. 51:1086-1092.

4. Hirano, M., et al. 2001. Defects of intergenomic communication: autosomal disorders that cause multiple deletions and depletion of mitochondrial DNA. Semin. Cell Dev. Biol. 12:417-427.

5. Hirano, M., et al. 1998. Mitochondrial neurogastrointestinal encephalomyopathy syndrome maps to chromosome 22q13.32-qter. Am. J. Hum. Genet. 63:526-533.

6. Nishino, I., Spinazzola, A., and Hirano, M. 1999. Thymidine phosphorylase gene mutations in MNGIE, a human mitochondrial disorder. Science. 283:689-692.

7. Spinazzola, A., et al. 2002. Altered thymidine metabolism due to defects of thymidine phosphorylase. J. Biol. Chem. 277:4128-4133.

8. Trounce, I.A., Kim, Y.L., Jun, A.S., and Wallace, D.C. 1996. Assessment of mitochondrial oxidative phosphorylation in patient muscle biopsies, lymphoblasts, and transmitochondrial cell lines. Methods Enzymol. 264:484-509.

9. Bradford, M.M. 1976. A rapid and sensitive method for the quantitation of microgram quantities of protein utilizing the principle of protein-dye binding. Anal. Biochem. 72:248-254.

10. Hartwick, R.A., Assenza, S.P., and Brown, P.R. 1979. Identification and quantitation of nucleosides, bases and other UV-absorbing compounds in serum, using reversed-phase high-performance liquid chromatography. I. Chromatographic methodology. J. Chromatogr. 186:647-658.

11. Hartwick, R.A., Krstulovic, A.M., and Brown, P.R. 1979. Identification and quantitation of nucleosides, bases and other UV-absorbing compounds in serum, using reversed-phase high-performance liquid chromatography. II. Evaluation of human sera. J. Chromatogr. 186:659-676.

12. Nishigaki, Y., et al. 2002. Exercise-induced muscle "burning," fatigue, and hyper-CKemia: mtDNA T10010C mutation in tRNA(Gly). Neurology. 58:1282-1285

13. Parsons, T.J., et al. 1997. A high observed substitution rate in the human mitochondrial DNA control region. Nat. Genet. 15:363-368.

14. Stoneking, M. 2000. Hypervariable sites in the mtDNA control region are mutational hotspots. Am. J. Hum. Genet. 67:1029-1032.

15. Malyarchuk, B.A., Rogozin, I.B., Berikov, V.B., and Derenko, M.V. 2002. Analysis of phylogenetically reconstructed mutational spectra in human mitochondrial DNA control region. Hum. Genet. 111:46-53.

16. Anderson, S., et al. 1981. Sequence and organization of the human mitochondrial genome. Nature. 290:457-465.

17. Spagnolo, M., et al. 2001. A new mutation in the mitochondrial tRNA(Ala) gene in a patient with ophthalmoplegia and dysphagia. Neuromuscul. Disord. 11:481-484.

18. Manfredi, G., et al. 1996. Identification of a mutation in the mitochondrial tRNA(Cys) gene associated with mitochondrial encephalopathy. Hum. Mutat. 7:158-163.

19. Santorelli, F.M., et al. 1997. Mitochondrial tRNA(Cys) gene mutation
(A5814G): a second family with mitochondrial encephalopathy. Neuromuscul. Disord. 7:156-159.

20. Karadimas, C., et al. 2001. A5814G mutation in mitochondrial DNA can cause mitochondrial myopathy and cardiomyopathy. J. Child Neurol. 16:531-533.

21. McCallum, F.S., and Maden, B.E. 1985. Human 18 S ribosomal RNA sequence inferred from DNA sequence. Variations in $18 \mathrm{~S}$ sequences and secondary modification patterns between vertebrates. Biochem. J. 232:725-733.

22. Nakajima-Iijima, S., Hamada, H., Reddy, P., and Kakunaga, T. 1985. Molecular structure of the human cytoplasmic beta-actin gene: interspecies homology of sequences in the introns. Proc. Natl. Acad. Sci. U. S. A. 82:6133-6137.

23. Bestwick, R.K., and Mathews, C.K. 1982. Unusual compartmentation of precursors for nuclear and mitochondrial DNA in mouse L cells. J. Biol. Chem. 257:9305-9308.

24. Bestwick, R.K., Moffett, G.L., and Mathews, C.K. 1982. Selective expansion of mitochondrial nucleoside triphosphate pools in antimetabolitetreated HeLa cells. J. Biol. Chem. 257:9300-9304.

25. Berk, A.J., and Clayton, D.A. 1973. A genetically distinct thymidine kinase in mammalian mitochondria. Exclusive labeling of mitochondrial deoxyribonucleic acid. J. Biol. Chem. 248:2722-2729.

26. Bogenhagen, D., and Clayton, D.A. 1976. Thymidylate nucleotide supply for mitochondrial DNA synthesis in mouse L-cells. Effect of 5-fluorodeoxyuridine and methotrexate in thymidine kinase plus and thymidine kinase minus cells. J. Biol. Chem. 251:2938-2944.

27. Croteau, D.L., Stierum, R.H., and Bohr, V.A. 1999. Mitochondrial DNA repair pathways. Mutat. Res. 434:137-148.

28. Phear, G., Nalbantoglu, J., and Meuth, M. 1987. Next-nucleotide effects in mutations driven by DNA precursor pool imbalances at the aprt locus of Chinese hamster ovary cells. Proc. Natl. Acad. Sci. U. S. A. 84:4450-4454.

29. Kunkel, T.A., and Alexander, P.S. 1986. The base substitution fidelity of eucaryotic DNA polymerases. Mispairing frequencies, site preferences, insertion preferences, and base substitution by dislocation. J. Biol. Chem. 261:160-166.

30. Longley, M.J., Nguyen, D., Kunkel, T.A., and Copeland, W.C. 2001. The fidelity of human DNA polymerase gamma with and without exonucleolytic proofreading and the p55 accessory subunit.J. Biol. Chem. 276:38555-38562.

31. Johnson, A.A., and Johnson, K.A. 2001. Fidelity of nucleotide incorporation by human mitochondrial DNA polymerase. J. Biol. Chem. 276:38090-38096.

32. Fersht, A.R. 1979. Fidelity of replication of phage phi X174 DNA by DNA polymerase III holoenzyme: spontaneous mutation by misincorporation. Proc. Natl. Acad. Sci. U. S. A. 76:4946-4950.

33. Kunkel, T.A., and Soni, A. 1988. Mutagenesis by transient misalignment. J. Biol. Chem. 263:14784-14789.

34. Lim, S.E., and Copeland, W.C. 2001. Differential incorporation and removal of antiviral deoxynucleotides by human DNA polymerase gamma. J. Biol. Chem. 276:23616-23623.

35. Grollman, A.P., and Moriya, M. 1993. Mutagenesis by 8-oxoguanine: an enemy within. Trends Genet. 9:246-249.

36. Eftedal, I., Guddal, P.H., Slupphaug, G., Volden, G., and Krokan, H.E. 1993. Consensus sequences for good and poor removal of uracil from double stranded DNA by uracil-DNA glycosylase. Nucleic Acids Res. 21:2095-2101.

37. Moslemi, A.R., Melberg, A., Holme, E., and Oldfors, A. 1996. Clonal expansion of mitochondrial DNA with multiple deletions in autosomal dominant progressive external ophthalmoplegia. Ann. Neurol. 40:707-713.

38. Moslemi, A.R., Lindberg, C., and Oldfors, A. 1997. Analysis of multiple mitochondrial DNA deletions in inclusion body myositis. Hum. Mutat. 10:381-386.

39. Carrozzo, R., et al. 1998. Multiple mtDNA deletions features in autosomal dominant and recessive diseases suggest distinct pathogeneses. Neurology. 50:99-106.

40. Shadel, G.S., and Clayton, D.A. 1997. Mitochondrial DNA maintenance in vertebrates. Annu. Rev. Biochem. 66:409-435.

41. Rampazzo, C., et al. 2000. A deoxyribonucleotidase in mitochondria: involvement in regulation of dNTP pools and possible link to genetic disease. Proc. Natl. Acad. Sci. U. S. A. 97:8239-8244. 